\title{
Inappropriate Use of Nonpsychotropic Medications in Nursing Homes
}

\author{
Brent Williams, MD, MPH* and Charles Betley, $M A^{\dagger}$
}

OBJECTIVES: To determine the prevalence and patientspecific predictors of the use of 10 presumptively inappropriate medications used to treat medical conditions among nursing home residents, and to use this information to examine alternative screening strategies using computerized assessment data to identify residents who are at high risk of receiving inappropriate medications.

DESIGN: Retrospective, cross-sectional study.

PATIENTS: All persons residing in all 252 nursing homes in two states during the last 6 months of $1991(\mathrm{~N}=21,884)$.

MEASUREMENTS: Data were from Minimum Data Set Plus (MDS+) assessments, gathered as part of the Health Care Financing Administration (HCFA) Multistate Nursing Home Casemix and Quality Demonstration Project. The MDS + is an expanded version of the federally mandated Minimum Data Set (MDS) that includes additional information on medications and their doses and schedules (frequency, standing vs prn). The reliability of the MDS has been demonstrated previously. Medications were defined as inappropriate using explicit criteria from published literature.

Outcome measures were the standing use of each or any of 10 presumptively inappropriate medications used to treat medical (rather than psychiatric or behavioral) conditions. Potential predictors of inappropriate medication use included patient demographic characteristics, payer, a proxy measure for length of stay and admission source, functional status, number of standing medications, and state.

MAIN RESULTS: A total of $12 \%$ of residents were prescribed one or more of 10 presumptively inappropriate medications on a standing basis, a figure that differed substantially between states $(14.0 \%$ vs $7.4 \%(P<.001))$. The most prevalent inappropriate medications were dipyridamole (5.4\% of residents), amitriptyline $(3.3 \%)$, and methyldopa $(1.8 \%)$. Among parients receiving 0 to 3,4 to 6 , and $7+$ medications, $5 \%, 12 \%$, and $19 \%$, respectively, were receiving at least one inappropriate medication. In multivariate

From the "Department of Internal Medicine, University of Michigan, the Geriatrics Research Education and Clinical Center, Ann Arbor VA Medical Center, and the tDepartment of Health Services Management and Policy, University of Michigan, Ann Arbor, Michigan.

Supported, in part, by National Institute of Aging Grant AG08808-05 through the University of Michigan Geriatrics Center Pilot/Feasibility Grants Program. Portions presented at the 46th Annual Scientific Meeting of the Gerontological Sociery of America, New Orleans, Louisiana, November 1993.

Address correspondence to Brent Williams, MD, MPH, University of Michigan Medical Center, Dept. of Internal Medicine, Div. of General Medicine, 3116 Taubman Center, 1500 E. Medical Center Dr., Ann Arbor, MI 48109-0376. logistic regression analyses, the strongest predictors of inappropriate medication use were state and the total number of standing medications prescribed. Including other statistically significant predictors of inappropriate medication use (age $>$ 65 years, never having been married, severe functional limitations, being a long-stay patient, and medical diagnosis) did not substantially improve the overall predictive ability of the model.

CONCLUSIONS: A substantial proportion of nursing home residents receives presumptively inappropriate medications to treat medical conditions. Selecting persons prescribed large numbers of medications for further review may be the most efficient method for nursing home or pharmacy personnel to identify residents at high risk of receiving inappropriate medications. Extensive additional information on residents' characteristics, although widely available through the Minimum Data Set, does not significantly improve the ability to identify residents receiving inappropriate medications for medical conditions. State-specific policies or provider practices also influence the likelihood of presumptively inappropriate medication use among nursing home residents and deserve further investigation. J Am Geriatr Soc 43:513-519, 1995.

$\mathrm{P}$ hysician care provided to nursing home residents has been criticized as sporadic, poorly documented, and inadequate to meet residents' medical needs. ${ }^{1}$ One aspect of the medical care of nursing home residents-the use of sedative/ hypnotic and antipsychotic and antidepressant (collectively termed psychotropic) medications in the treatment of behavioral and psychological disorders-has received much attention in recent years. ${ }^{2,3}$ Studies have found widespread overuse of psychotropic medications in nursing homes, ${ }^{2,4-6}$ poor documentation of medical diagnoses for which psychotropic medications are indicated, ${ }^{2,7}$ and positive effects of interventions designed to decrease the use of psychotropic medications among nursing home residents. ${ }^{8}$ Partly in response to these findings, the use of antipsychotic medications among nursing home residents has been the subject of recent federal legislation to improve the quality of nursing home care.

Although psychotropic medications prescribed nursing home residents have received much attention, relatively little attention has been paid to the appropriateness of medications used to treat medical conditions in nursing homes. Since nursing home residents commonly receive large numbers of medications, many of which are nonpsychotropic, ${ }^{5,9}$ examining nonpsychotropic medication use in this population may be one method to improve the quality of medical care in nursing homes. Identifying instances of inappropriate non- 
psychotropic medication use among nursing home residents has been difficult, however, because (1) judging whether a medical regimen is appropriate for a given patient often requires detailed knowledge of the patient's medical history, which is often lacking in nursing home records, and (2) even with perfect medical knowledge about a given patient, widely accepted criteria defining appropriate medications have not been developed.

Substantial progress in developing methods to identify instances of inappropriate medication use was made by Beers and colleagues, ${ }^{10}$ who developed, through expert panel consensus, a list of approximately 30 medications that are presumptively inappropriate for nursing home residents. Drugs (or drug dosages) were defined as inappropriate if equally effective alternative medications were available that had substantially lower risk of adverse effects (e.g., chlorpropamide for adult-onset diabetes mellitus) or if scientific evidence was insufficient to support the use of the medication under any circumstances (e.g., isoxsuprine for dementia). One intended advantage of these criteria was that any instance of the use of the medications (or medication dosages) could be defined as (presumptively) inappropriate, allowing the use of computerized databases, which often lack detailed clinical information, to screen for their use among large groups of nursing home residents.

The purposes of this study were to identify the prevalence and associated patient characteristics of presumptively inappropriate nonpsychotropic medication use among nursing home residents from two states. Study goals were (1) to estimate the "yield" of screening patients' medication profiles via computer for inappropriate medications as one potential component of a quality assurance system, and (2) to determine whether information about patients' demographic, clinical, and functional characteristics and medical diagnoses could be used to identify residents at highest risk of receiving inappropriate medications for medical conditions, whose medication profiles could then be prioritized for review. This latter goal was important to determine whether patients at high risk for receiving inappropriate medications could be identified using detailed resident information in settings where computerized medication information is not available. With the widespread implementation of federally mandated uniform resident assessments in nursing homes, the ability to identify patients at high risk of suboptimal care and to target these patients for further review could substantially improve the efficiency of quality review efforts.

\section{METHODS}

\section{Data}

Data were collected as part of the Health Care Financing Administration's Multistate Nursing Home Casemix and Quality (NHCMQ) Demonstration Project. The purposes of the larger project are to develop and implement a case-mix payment system (Resource Utilization Groups, Version III [RUG-III]) ${ }^{11}$ and to develop methods to assess quality of care in nursing homes. Data for the HCFA Multistate NHCMQ Demonstration Project were gathered using an expanded version of the Minimum Data Set (MDS), the Minimum Data Set Plus (MDS+). The MDS+ was administered to all nursing home residents of four states that were centrally involved in the NHCMQ Demonstration Project and in several states with adjunctive relationships to the project. Implementation of the MDS+ began in the four core states in October 1990. By July 1, 1991, two of the four states were collecting and computerizing data about all residents. Data from these two states were used in this study.

The MDS is a federally mandated assessment instrument implemented in nursing homes throughout the United States that includes extensive information about patient demographic characteristics, functional status, and nursing needs relevant to care planning. The development of the MDS has been described in more detail elsewhere, ${ }^{12}$ and the high reliability of MDS items has been demonstrated. ${ }^{12-13}$ For example, among residents of 13 nursing homes in five states the interrater reliability (Spearman-Brown intraclass correlation coefficient) was 0.7 or higher for all items measuring activities of daily living (ADL) performance, cognition, continence, and total number of medications. ${ }^{13}$ The MDS+ includes all items from the MDS, as well as several additional items, including detailed information on all medications used by residents. Medication information included type of medication, dosage, frequency of administration, and whether standing or prn (pro re nata, as necessary). The reliability of the detailed medication information available in the MDS+ has not been formally determined. Nursing homes were allowed flexibility in choosing the person or persons to complete the MDS+ assessments. Commonly, the assessor was either the resident's primary nurse or a multidisciplinary team of persons with direct responsibility for care of the resident. Active medical problems (explicitly defined as conditions that have a relationship to the resident's functional status, cognitive status, behavioral status, medical treatments, or risk of death) were determined by a registered nurse from the medical record and listed on the MDS + form, either in a check-box section containing 31 specific diagnoses or a fill-in section for additional diagnoses. Interrater reliabilities for check-box diagnoses have been demonstrated to be high, (e.g., $0.65,0.69$, and 0.80 for depression, stroke, and hypertension, respectively). ${ }^{13}$ However, it is unknown whether the implementation of the MDS has affected underreporting of medical diagnoses, which has been demonstrated among nursing home residents. ${ }^{2,7}$

Assessments were carried out within 2 weeks of admission or readmission to the nursing home, after a significant change in clinical status, and no less often than quarterly.

Data for the study were taken from the most recent MDS+ assessment for each nursing home resident in two states during the period July 1, 1991, to December 31, 1991. Data were obtained from 21,884 residents from 252 nursing homes. Thus the study population included all patients residing in nursing homes as well as all patients admitted (or readmitted) to nursing homes of the two states during the last 6 months of 1991. Approximately three-quarters of the assessments $(n=15,107)$ were from one state (hereafter, State $A)$, and one-quarter $(n=6777)$ were from the second state (hereafter, State B). Both states requested that states' identities remain confidential.

Data were largely complete and internally consistent. For approximately $5 \%$ of residents, demographic data (age, race, and gender) and admission source (hospital, home, other nursing home, other source) were missing. The proportion of assessments with missing data was different between the states; approximately $12 \%$ of assessments in the smaller state (State B) were missing demographic and admission source information, compared with approximately $2 \%$ of assess- 
ments in the larger state (State A). For other data elements, data were missing for no more than $2 \%$ of assessments in either state.

\section{Inappropriate Medications}

Of the 30 inappropriate medications identified by Beers et al., ${ }^{10} 10$ were included in the present study. Excluded were: (1) anxiolytics and major tranquilizers (psychotropic medications), and (2) medications likely to be used on a prn basis (e.g., antispasmodics). The latter criteria was included because we wished to focus on physician rather than nursing behavior and to elucidate relationships between specific medical conditions and physician prescribing patterns. Amitriptyline (a psychotropic medication) was included because, unlike other psychotropic medications, it is commonly used to treat two common medical conditions- urinary incontinence and peripheral neuropathy. Information on psychotropic medications (other than amitriptyline) was not included in the data made available for these analyses.

\section{Predictor Variables}

\section{Resident Demographic Characteristics and Functional Status}

Functional status was summarized according to RUG-III ADL Index groups. ${ }^{11}$ This ADL scale combines information on patients' capacity to move around in bed, eat, transfer from bed to chair, and get on and off a toilet. The resulting score ranges from 4 to 18 , with higher scores representing increasing levels of impairment. Patients were grouped according to scores $4-7,8-15$, and 16-18 inasmuch as nursing home residents within these categories have been shown to be relatively homogeneous with respect to the time spent by nursing staff in caring for them. ${ }^{11}$ In explaining nursing time spent in patient-specific activities, the RUG-III ADL Index performed comparably to the Katz and Barthel ADL Indexes. ${ }^{14}$

\section{Primary Payer}

Reimbursement for prescription drugs differs substantially among payers. For example, Medicaid covers prescription drugs, whereas some payers do not. Because the presence of a drug benefit has been shown to substantially affect prescribing practices, ${ }^{15}$ the relationship of payer and inappropriate medication use was examined.

\section{Type of Assessment}

It was important to distinguish inappropriate medication use that reflected prescribing patterns in the nursing home rather than the residents' environment before admission (most often hospital or home). Residents were classified first by the listed indication for the assessment-admission/ readmission versus "routine" (quarterly or annual assessment) - to distinguish recently admitted residents from longstayers. Admitted/readmitted residents (assessed within 14 days of admission) were then further classified by source of admission-hospital, home, or nursing home-to identify potential differences in prescribing patterns among these settings. (Direct information on length of stay, although coded in the MDS+, could not be used because of internal inconsistencies in the data for approximately $10 \%$ of residents. It appeared, for example, that among patients who were hospitalized during their nursing home stay, the date of admission to the nursing home was variably coded as the date of original admission or date of readmission from the hospital).

\section{Number of Standing Medications}

We wished to determine whether the prescription of large numbers of medications per resident was disproportionately associated with the receipt of inappropriate medications. This type of association would suggest that receiving large numbers of medications is a marker for poor prescribing practices in general, including prescription of inappropriate medications. Alternatively, if receipt of inappropriate medications was proportionate to the total number of medications, this would indicate an exposure effect, whereby patients prescribed large numbers of medications are at higher risk of inappropriate medications as a result of the large number of medications per se rather than because of uniquely poor prescribing practices.

\section{Medical Diagnosis}

Although it is known that medical diagnoses of nursing home residents are generally underreported in medical records, ${ }^{7}$ we wished to examine the feasibility of using medical diagnoses from nursing home medical records (recorded in the MDS+) to identify patients receiving inappropriate medications in settings in which detailed medication information is not readily available for quality review.

\section{Statistical Methods}

Bivariate relationships between receipt of inappropriate medications and patient characteristics were examined using the chi-square test for homogeneity. Multivariate predictors of the use of any inappropriate medications were determined using logistic regression analyses. In multivariate models, demographic variables (age, race, gender, marital status) and payer were included in all models. Other variables were retained only if they were statistically significant $(P<.05)$.

The sensitivity of the findings to several factors, particularly for the multivariate models, was examined in several ways. To assess the effects of missing data on the multivariate models, observations with missing data were alternatively included in the reference category for that variable and excluded from the model. Potential clustering effects attributable to relatively few practitioners prescribing medications in each nursing home was examined using the Huber method to adjust the standard error estimates in the multivariate models. ${ }^{16}$ Assumptions of logistic regression were examined using the Hosmer-Lemeshow test. ${ }^{17}$

Statistical analyses were carried out using SAS/PC ${ }^{18}$ and STATA. ${ }^{19}$

\section{RESULTS}

The study population was similar to other nursing home populations in the United States ${ }^{20}$ (Table 1). The mean (SD) age was $82.4(11.1)$ years, $73 \%$ were female, $16 \%$ were black, and $65 \%$ were widowed. The most important differences in resident characteristics between states were that $23 \%$ of the residents in State $A$ (the larger state) were black compared with $<1 \%$ in State $B(P<.001)$, and Medicaid was primary payor for $78 \%$ of residents in State A versus $47 \%$ in State $B(P<.001)$. The prevalence of functional limitations was high, with $34 \%$ of residents requiring active (hands-on) human help with eating, $45 \%$ with moving around in bed, and $65 \%$ with toileting. A total of $45 \%$ percent of residents 
Table 1. Characteristics of Nursing Home Residents in Two States

\begin{tabular}{|c|c|}
\hline Variable & $\begin{array}{c}\text { Percent of } \\
\text { Residents } \\
(N=21,884)^{\prime}\end{array}$ \\
\hline \multicolumn{2}{|l|}{ Age (Years) } \\
\hline$<65$ & 6.7 \\
\hline $65-74$ & 12.7 \\
\hline $75-84$ & 35.0 \\
\hline $85-94$ & 37.8 \\
\hline $95+$ & 7.7 \\
\hline \multicolumn{2}{|l|}{ Gender } \\
\hline Male & 26.8 \\
\hline Female & 73.2 \\
\hline \multicolumn{2}{|l|}{ Race } \\
\hline White & 82.3 \\
\hline Black & 16.4 \\
\hline Other & 1.4 \\
\hline \multicolumn{2}{|l|}{ Marital Status } \\
\hline Married & 15.5 \\
\hline Single & 14.1 \\
\hline Separated, widowed, divorced & 70.4 \\
\hline \multicolumn{2}{|l|}{$\begin{array}{l}\text { Requires active human assistance to } \\
\text { (may have more than one) }\end{array}$} \\
\hline Eat & 34.3 \\
\hline Move around in bed & 45.2 \\
\hline Toilet & 64.9 \\
\hline Bladder incontinence & 44.8 \\
\hline \multicolumn{2}{|l|}{ Number of standing medications } \\
\hline $0-3$ & 31.5 \\
\hline $4-6$ & 37.5 \\
\hline $7-9$ & 21.5 \\
\hline $10+$ & 9.5 \\
\hline \multicolumn{2}{|l|}{ Type of assessment ${ }^{* *}$} \\
\hline Admission from hospital & 13.3 \\
\hline $\begin{array}{l}\text { Admission from home or nursing } \\
\text { home }\end{array}$ & 6.6 \\
\hline Quarterly assessment & 80.1 \\
\hline \multicolumn{2}{|l|}{ Payer (may have more than one) } \\
\hline Self/private insurance & 47.2 \\
\hline Medicare & 4.7 \\
\hline Medicaid & 63.1 \\
\hline \multicolumn{2}{|l|}{$\begin{array}{l}\text { Selected medical diagnoses (may } \\
\text { have more than one) }\end{array}$} \\
\hline Depression & 9.3 \\
\hline Peripheral neuropathy & 0.4 \\
\hline Stroke & 25.0 \\
\hline Hypertension & 33.6 \\
\hline \multicolumn{2}{|l|}{$\begin{array}{l}\text { Number of inappropriate standing } \\
\text { medications }\end{array}$} \\
\hline None & 88.1 \\
\hline One & 11.2 \\
\hline Two or three & 0.7 \\
\hline
\end{tabular}

* Percentages exclude missing values; see text for details.

* * See text for definitions.

were incontinent of bladder at least twice a week. The mean (SD) number of standing (non-prn) medications prescribed was 5.2 (3.1), a value that did not vary between the states. Approximately $20 \%$ of patients had admission/readmission assessments and, thus, had resided in the nursing home less than 2 weeks. Of these, roughly three-quarters were admitted/readmitted from the hospital.

A total of $12 \%$ of residents received at least one of 10 inappropriate medications (Table 2). By far the most common inappropriate medications were dipyridamole, amitriptyline, and methyldopa. Each of the remaining seven medications or medication dosages was prescribed for less than $1 \%$ of residents. The large majority of patients receiving any inappropriate medications received only one, with only $0.7 \%$ of patients receiving two or three inappropriate medications (Table 1).

Differences in the proportions of residents in the two states who were prescribed each inappropriate medication or any inappropriate medication were substantial. For example, proportionately twice as many residents of State A were prescribed inappropriate medications compared with State B ( 14 vs $7 \%, P<.001)$. However, the relative prevalence of inappropriate medications was similar between the states, with dipyridamole, amitriptyline, and methyldopa the three most common inappropriate medications in both states.

\section{Total Number of Standing Medications and the Use of Inappropriate Medications}

The likelihood of receiving any inappropriate medication was strongly positively associated with increasing numbers of standing medications (Figure 1). For example, only $5.4 \%$ of patients receiving 0 to 3 standing medications $(32 \%$ of the population) were receiving at least one inappropriate medication, whereas $22.3 \%$ of persons receiving 10 or more medications $(10 \%$ of the population) were receiving at least one inappropriate medication.

\section{Medical Diagnoses and the Use of Inappropriate Medications}

The use of inappropriate medications was associated with the documented presence of specific medical diagnoses. Among the 1181 patients receiving dipyridamole, 581 (49\%) had a documented diagnosis of stroke (cerebrovascular accident or transient ischemic attack); of the 396 patients receiving methyldopa, $317(80 \%)$ had a diagnosis of hypertension; and of the 714 patients prescribed amitriptyline, $444(62 \%)$

Table 2. Prevalence of Presumptively Inappropriate Medications Among Nursing Home Residents

\begin{tabular}{lc}
\hline \multicolumn{1}{c}{ Medication } & $\begin{array}{c}\text { Percent of } \\
\text { Residents } \\
(\mathrm{N}=\mathbf{2 1 , 8 8 4})\end{array}$ \\
\hline Dipyridamole & 5.4 \\
Amitriptyline & 3.3 \\
Methyldopa & 1.8 \\
Reserpine & 0.8 \\
Chlorpropamide & 0.5 \\
Cimetidine $>$ 900 mg/day & 0.4 \\
Hydrochlorothiazide $>50$ mg/day & 0.1 \\
Ranitidine $>$ 300 mg/day & 0.1 \\
Cyclandelate & 0.1 \\
Isoxsuprine & 0.1 \\
Any inappropriate medication & 12.0 \\
\hline
\end{tabular}




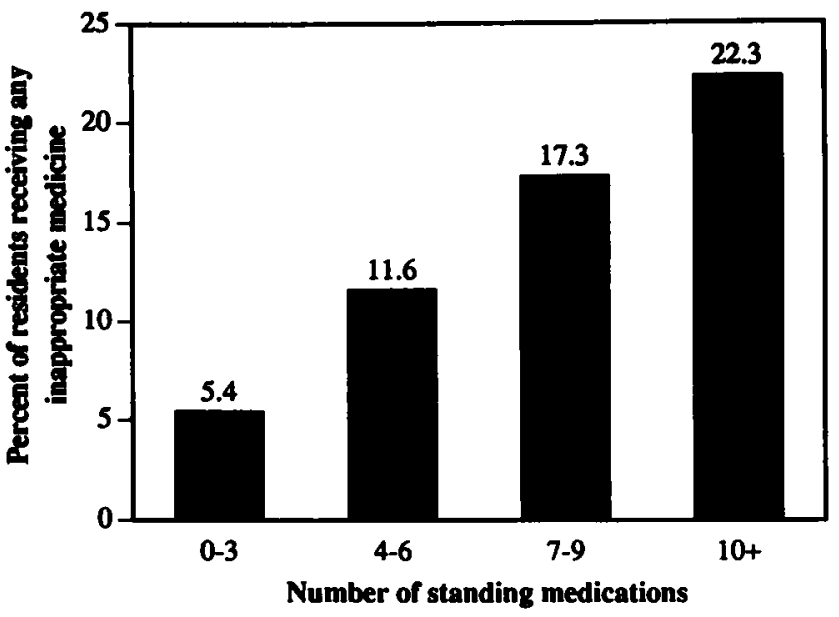

Figure 1. Relationship between receipt of any inappropriate medication and number of standing medications $(\mathrm{N}=21,884)$.

had a diagnosis of depression, peripheral neuropathy, or were incontinent of urine.

Conversely, the presence of selected medical diagnoses was not a very efficient method for identifying the use of inappropriate medications. For example, only $10 \%$ of patients with the documented diagnosis of stroke received dipyridamole; only $4 \%$ of patients with the diagnosis of hypertension received methyldopa; and only $3 \%$ of patients with the diagnosis of depression, peripheral neuropathy, or urinary incontinence received amitriptyline.

\section{Multivariate Models Predicting Use of Any Inappropriate Medication}

The strongest bivariate predictors-total number of standing medications and state-persisted in multivariate models that controlled for patient age, gender, race, marital status, source of admission, payer, and functional status (Table 3).

There was a generally linear relationship between the total number of standing medications and the likelihood of receiving an inappropriate medication, though this effect leveled off somewhat at around 10 to 12 medications in multivariate models. Since only $10 \%$ of patients received 10 or more medications, patients with seven or more standing medications were classified together in multivariate models. The odds of receiving at least one inappropriate medication among persons receiving 4 to 6 or 7 or more standing medications were 2.2 and 3.8 times higher, respectively, than among persons receiving 0 to 3 standing medications.

State remained a strong predictor of the use of any inappropriate medication, with residents of State B only half as likely as residents of State $A$ to receive at least one inappropriate medication.

Receiving one or more inappropriate medication was also associated with age greater than 65 , female gender, being single (never married), having a quarterly assessment (a proxy for long nursing home stay), and having severe functional limitations. Black race was negatively and receipt of Medicaid was positively associated with inappropriate medication use, but these associations were not statistically significant.
Table 3. Logistic Regression Model Predicting Receipt of Any Inappropriate Medication

\begin{tabular}{|c|c|c|}
\hline Predictors & $\begin{array}{l}\text { Odds } \\
\text { Ratio }\end{array}$ & $\begin{array}{l}\text { (95\% Conf. } \\
\text { Interval) }\end{array}$ \\
\hline \multicolumn{3}{|l|}{ Age } \\
\hline Age less than 65 & 0.75 & $(0.60,0.94)$ \\
\hline Age $65-84$ & 1.00 & \\
\hline Age 85 or greater & 0.94 & $(0.85,1.03)$ \\
\hline \multicolumn{3}{|l|}{ Gender } \\
\hline Female & 1.00 & \\
\hline Male & 0.90 & $(0.80,1.01)$ \\
\hline \multicolumn{3}{|l|}{ Race } \\
\hline White & 1.00 & \\
\hline Black & 0.90 & $(0.79,1.04)$ \\
\hline Other, non-black & 0.96 & $(0.62,1.46)$ \\
\hline \multicolumn{3}{|l|}{ Marital status } \\
\hline Widowed, separated, divorced & 1.00 & \\
\hline Married & 1.00 & $(0.88,1.16)$ \\
\hline Never married & 0.82 & $(0.70,0.96)$ \\
\hline \multicolumn{3}{|l|}{ Type of assessment } \\
\hline Admission from hospital & 1.00 & \\
\hline $\begin{array}{l}\text { Admission from home or nursing } \\
\text { home }\end{array}$ & 1.01 & $(0.79,1.29)$ \\
\hline Quarterly assessment & 1.15 & $(0.99,1.33)$ \\
\hline \multicolumn{3}{|l|}{ Payment source } \\
\hline \multicolumn{3}{|l|}{ Medicaid payment } \\
\hline No & 1.00 & \\
\hline Yes & 1.09 & $(0.96,1.23)$ \\
\hline \multicolumn{3}{|l|}{ Self-pay or private insurance } \\
\hline No & 1.00 & \\
\hline Yes & 1.01 & $(0.91,1.12)$ \\
\hline \multicolumn{3}{|l|}{ Medicare payment } \\
\hline No & 1.00 & \\
\hline Yes & 1.17 & $(0.94,1.45)$ \\
\hline \multicolumn{3}{|l|}{ Standing medications } \\
\hline 3 or fewer & 1.00 & \\
\hline $4-6$ & 2.18 & $(1.90,2.50)$ \\
\hline 7 or more & 3.81 & $(3.30,4.40)$ \\
\hline \multicolumn{3}{|l|}{ RUG-III ADL Index* } \\
\hline $4-7$ & 1.00 & \\
\hline $8-15$ & 1.00 & $(0.90,1.10)$ \\
\hline 16 or more & 0.82 & $(0.71,0.95)$ \\
\hline \multicolumn{3}{|l|}{ State } \\
\hline State $A$ & 1.00 & \\
\hline State B & 0.48 & $(0.43,0.54)$ \\
\hline
\end{tabular}

Number of obs $=17,661$

Log likelihood $=-6149.86$

$\chi^{2}(17)=703.29$

Pseudo $R^{2}=0.054$

*RUG-III is Resource Utilization Groups, version 3. See text for definition; higher levels denote greater functional limitation. Includes information on bed mobility, eating, transferring, and toileting.

Interaction terms were examined among demographic characteristics, primary payer, and functional status. None was significant, except for being black and on Medicaid, which were associated with increased likelihood of receiving inappropriate medications $(P=.04)$. Inclusion of this term in the multivariate model did not substantially affect the remaining odds ratios in the model. 
The multivariate model had a high sensitivity but low specificity for identifying persons receiving inappropriate medications. For example, using a predicted probability of 0.1 as the cutoff, $75 \%$ of persons with and $53 \%$ of persons without inappropriate medications were correctly identified by the full model. This was comparable to the performance of a restricted model, which included only information on the number of standing medications and state, that correctly identified $79 \%$ of persons with and $46 \%$ of persons without inappropriate medications.

The main findings of the multivariate models were insensitive to whether patients with missing values were included in the reference categories for the relevant variables and to application of the Huber correction for cluster effects with nursing homes.

\section{DISCUSSION}

The prevalence of inappropriate medications used to treat medical conditions among nursing home residents in two states was substantial, with nearly one in eight residents receiving at least one of 10 selected inappropriate medications. This prevalence is consistent with that found by Beers and colleagues ${ }^{21}$ for the same medications in 12 nursing homes in California during the same period. However, Beers et al. reported the rate of inappropriate medication use as a percent of all prescriptions rather than percent of patients, making direct comparisons difficult. The total prevalence of inappropriate medication use is likely to be much higher in our study population, because psychotropic (other than amitriptyline) and prn medications were not included. With the prevalence of inappropriate medication use this high, screening all residents for the presence of one or more presumptively inappropriate medications in nursing homes with access to computerized medication profiles would result in a reasonable "hit rate." To make the system more efficient, or in nursing homes without access to computerized information on medications, patients prescribed large numbers (e.g., more than six standing or eight total medications) of medications could be targeted for review. In this population, among residents receiving seven or more standing medications ( $31 \%$ of residents), $19 \%$ received at least one inappropriate medication. Information on the total number of medications used by residents is recorded on the MDS. Using other information routinely available from MDS assessments among nursing home residents is unlikely to improve substantially the ability to identify residents at high risk for use of inappropriate medications for medical conditions.

Once patients receiving presumptively inappropriate medications are identified, several courses of action could be taken. The prescribing physician could be contacted with the recommendation that the medication be stopped or changed to a safer alternative. If resources permit, information from the medical literature could be provided as well to justify this recommendation. Physicians have responded positively to academic detailing of this type. ${ }^{8}$

A twofold difference in the prevalence of presumptively inappropriate medications between the two states was observed. This difference could be attributable to state-specific policies that affect prescribing patterns in nursing homes, regional differences in prescribing habits, or both. Future studies should investigate factors that could account for such large regional variations in the use of particular medications among nursing home residents.

One goal of the present study was to determine the usefulness of information on patient characteristics in identifying persons most likely to receive inappropriate medication. This seemed important because a large amount of detailed information is now widely available on nursing home residents, through the implementation of the MDS, and is becoming increasingly computerized, whereas detailed information on medications is not uniformly readily available for central review. After accounting for the total number of medications and state, including information on other patient characteristics did not significantly improve the ability to identify persons receiving inappropriate medications. This substantially simplifies the use of MDS information (which includes information on the total number of medications) in identifying patients who are most likely to benefit from detailed medication review.

The mean (SD) number of standing medications in the two states examined was $5.2(3.1)$, which is similar to other nursing home populations described over the past 2 decades. ${ }^{5,9}$ Thus, despite extensive documentation of the extent of polypharmacy among nursing home residents, the problem remains significant.

A definite but imperfect relationship between relevant medical diagnoses and the use of inappropriate medications was present in the study population. That is, a substantial proportion $(20-40 \%)$ of patients receiving inappropriate medications did not have medical diagnoses associated with the medication. This was best demonstrated in the case of methyldopa. Fully $20 \%$ of persons receiving methyldopa did not have a diagnosis of hypertension recorded in the medical record (as reflected in the MDS +), yet treatment of hypertension is the only medical indication for this medication. Thus, it is likely that the problem of underreporting medical conditions in the nursing home medical record persists and that diagnostic information is of little value in identifying residents at high risk of receiving inappropriate medications.

The associations of inappropriate medication use and other patient characteristics were generally similar to the positive associations reported between psychotropic medications in nursing homes and advanced age $e^{9,21-22}$ and female gender ${ }^{9,21}$ and the lack of association between receipt of Medicaid and inappropriate medication use. ${ }^{21}$

A strength of the present study is its focus on standing rather than prn medications. Most previous studies have not made this distinction, ${ }^{8,21}$ which has made it difficult to separate physician from nursing behavior in administration of inappropriate medications in nursing homes. The medications examined in the present study are prescribed by physicians only on a standing basis. Therefore, any actual use is solely the responsibility of the physician.

The primary limitation of this study is that the mere presence of a presumptively inappropriate medication does not necessarily demonstrate that suboptimal care is being provided or that adverse clinical outcomes are likely. One way to overcome this problem would be to focus on medications with no scientifically demonstrated benefit. Such unproven medications may have the greatest potential for use in identifying persons receiving poor quality medical care. However, of the medications examined here that have no scientifically demonstrated benefit (dipyridamole, cyclande- 
late, and isoxsuprine), only one (dipyridamole) occurred among more than $0.10 \%$ of patients. In addition, to our knowledge, the incidence of adverse clinical consequences associated with the use of dipyridamole among nursing home residents has not been demonstrated.

Other medications, which have proven benefit but high toxicity profiles relative to alternatives, may not, in fact, be inappropriate in every case (e.g., methyldopa at low doses, reserpine $^{23}$ ). At present, however, few explicitly derived criteria exist for defining inappropriate medications among nursing home residents, and very little data exist linking the use of particular medications to adverse clinical outcomes. Until such data are available, the approach taken here may remain the most efficient way to identify for further review patients at risk for poor medication prescribing practices.

\section{ACKNOWLEDGMENTS}

The authors greatly appreciate the contributions of David Zimmerman and Richard Ross in data preparation; Brant Fries, Elizabeth Cornelius, and reviewers from the study states in providing comments on the manuscript; and Clare Weipert in preparing the manuscript.

\section{REFERENCES}

1. Kane RA. Assessing quality in nursing homes. Clin Geriatr Med 1988;4:655-666.

2. Institute of Medicine. Improving the quality of nursing home care. Washington, DC: National Academy Press, 1986.

3. Siu AL. The quality of medical care received by older persons. J Am Geriatr Soc 1987;35:1084-1091.

4. Ray WA, Federspiel CF, Schaffner W. A study of antipsychotic drug use in nursing homes: Epidemiologic evidence suggesting misuse. Am J Public Health $1980 ; 70: 485-491$.

5. Beers M, Avorn J, Soumerai SB, et al. Psychoactive medication use in intermediate-care facility residents. JAMA 1988;260:3016-3020.
6. Buck JA. Psychotropic drug practice in nursing homes. J Am Geriatr Soc 1988;36:409-418.

7. Miller MB, Elliott DF. Errors and omissions in diagnostic records on admission of patients to a nursing home. J Am Geriatr Soc 1976;24:108-116.

8. Gurwitz JH, Soumerai SB, Avorn J. Improving medication prescribing and utilization in the nursing home. J Am Geriatr Soc 1990;38:542-552.

9. Robers PA. Extent of medication use in U.S. long-term care facilities. Am J Hosp Pharm 1988; 45:93-100.

10. Beers $\mathrm{MH}$, Ouslander JG, Rollingher I et al. Explicit criteria for determining inappropriate medication use in nursing home residents. Arch Intern Med 1991;151:1825-1832.

11. Fries BE, Schneider DP, Foley WJ et al. Refining a case-mix measure for nursing homes: Resource Utilization Groups (RUG-III). Med Care 1994; 32:68-85.

12. Morris JN, Hawes $C$, Fries BE et al. Designing the resident assessment instrument for nursing homes. Gerontologist 1990;30:293-307.

13. Hawes C, Morris JN, Phillips CD et al. Reliability estimates for the Minimum Data Set for nursing home residents. Gerontologist, 1995, in press.

14. Williams BC, Fries BE, Foley WJ et al. Activities of daily living and costs in nursing homes. Health Care Finan Rev 1994;15:1-19.

15. Soumerai SB, Ross-Degnan D, Gortmaker S, Avorn J. Withdrawing payment for nonscientific drug therapy. Intended and unexpected effects of a largescale natural experiment. JAMA 1990;263:831-839.

16. Stata Reference Manual: Release 3, Vol. 2, 5th Ed. Santa Monica, CA: Computing Resource Center, 1992, pp 338-346.

17. Hosmer DW, Lemeshow S. Applied logistic regression. New York: John Wiley \& Sons, 1989.

18. SAS Release 6.03. Cary, NC: SAS Institute, Inc., 1988.

19. STATA Version 3.00. Santa Monica, CA: Computing Resource Center, 1992.

20. Hing E, Sekscenski E, Strahan G. The National Nursing Home Survey: 1985 summary for the United States. Vital Health Stat 1989;13:102.

21. Beers $M H$, Ouslander JG, Fingold SF et al. Inappropriate medication prescribing in skilled-nursing facilities. Ann Intern Med 1992;117: 684-689.

22. Ingman SR, Lawson IR, Pierpaoli PG et al. A survey of the prescribing and administration of drugs in a long-term care institution for the elderly. J Am Geriatr Soc 1975;23:309-316.

23. Lederle FA, Applegate WB, Grimm RH Jr. Reserpine and the medical marketplace. Arch Intern Med 1993;153:705-706. 